\title{
On the condition number of certain Rayleigh-Ritz-Galerkin matrices
}

\section{Bernard J. Omodei}

\begin{abstract}
Martin H. Schultz [BuzZ. Amer. Math. Soc. 76 (1970), 840-844] has investigated the spectral condition number of the Rayleigh-RitzGalerkin matrices that arise when normalized $B$-spline coordinate functions are used to approximate the solution of a class of linear, self-adjoint, elliptic boundary value problems in one dimension. This paper shows how results analogous to those of Schultz [op. cit.] can be established under weaker assumptions. We also extend the results to boundary value problems in higher dimensions.
\end{abstract}

We consider the following class of linear, self-adjoint, two-point boundary value problems:

$$
\text { (1) } \begin{aligned}
L[u(x)] \equiv \sum_{j=0}^{n}(-1)^{j} D^{j}\left[p_{j}(x) D^{j} u(x)\right] & =f(x), \\
& 0<x<1, f \in L^{2}[0,1], n \geq 1,
\end{aligned}
$$

with homogeneous Dirichlet boundary conditions

$$
D^{k} u(0)=D^{k} u(1)=0,0 \leq k \leq n-1 \text {. }
$$

Assume that $p_{j}(x), 0 \leq j \leq n$, are real-valued bounded measurable functions on $[0,1]$.

Let $W_{0}^{n, 2}[0,1]$ denote the completion of the set of all $c^{\infty}[0,1]$ functions having compact support in $(0,1)$, with respect to the Sobolev Received 17 June 1976. 
norm

$$
\|w\|_{W^{n, 2}} \equiv\left\{\sum_{j=0}^{n} \int_{0}^{1}\left[D^{j} w(x)\right]^{2} d x\right\}^{\frac{1}{2}}
$$

We assume that there exists a positive constant $K$ such that for all $w \in W_{0}^{n, 2}[0,1]$,

$$
K\|v\|_{L^{2}}^{2} \leq \int_{0}^{1}\left\{\sum_{j=0}^{n} p_{j}(x)\left[D^{j} w(x)\right]^{2}\right\} d x
$$

This assumption corresponds to the assumption that the differential operator $L$ is positive definite. Schultz [7] made the stronger assumption that, for all $w \in W_{0}^{n, 2}[0,1]$,

$$
K\|w\|_{W^{n, 2}}^{2} \leq \int_{0}^{1}\left\{\sum_{j=0}^{n} p_{j}(x)\left[D^{j} w(x)\right]^{2}\right\} d x .
$$

It can be shown that the problem (1)-(2) has a unique generalized solution and that the Rayleigh-Ritz method is applicable; see Omodei [6]. Let $\left\{\phi_{i}(x)\right\}_{i=1}^{m}$ be $m$ given linearly independent coordinate functions such that $\phi_{i} \in W_{0}^{n, 2}[0,1]$ for all $1 \leq i \leq m$. Let $s_{m}$ denote the approximating subspace spanned by $\left\{\phi_{i}\right\}_{i=1}^{m}$. We claim, without giving the derivation, that the Rayleigh-Ritz-Galerkin matrix $R \equiv\left(r_{i k}\right)$ for the problem (1)-(2) is given by

$$
r_{i k}=\int_{0}^{1}\left\{\sum_{j=0}^{n} p_{j}(x) D^{j} \phi_{k}(x) D^{j} \phi_{i}(x)\right\} d x, \quad I \leq i, k \leq m .
$$

We now introduce normalized $B$-spline coordinate functions. Following the construction of de Boor [1], for a positive integer $d$, the finite set of real numbers

$$
\pi: 0=x_{0}<x_{1} \leq x_{2} \leq \ldots \leq x_{N}<x_{N+1}=1
$$

is said to be a $(d+1)$-extended partition of $[0,1]$, if and only if $x_{k}<x_{k+d}$ for all $0 \leq k \leq N-d+1$; that is, if $f_{k}$ denotes the 
multiplicity of the knot $x_{k}$ in $\pi$, then $f_{k} \leq d$ for all $l \leq k \leq N$. Let $I \equiv\left\{0 \leq k \leq N \mid x_{k}<x_{k+1}\right\}$, and define

$$
\Delta \equiv \max _{k \in I}\left(x_{k+1}-x_{k}\right) \text { and } \delta \equiv \min _{k \in I}\left(x_{k+1}-x_{k}\right) \text {. }
$$

Let $S p_{0}(d, \pi)$ denote the extended spline space of all extended splines of degree $d$ on $\pi$ satisfying the boundary conditions (2); that is, $S p_{0}(d, \pi)$ consists of those real-valued functions on $[0,1]$ which satisfy the boundary conditions (2), reduce to a polynomial of degree less than or equal to $d$ on $\left[x_{k}, x_{k+1}\right]$ for all $k \in I$, and have $d-f_{k}$ continuous derivatives in a neighbourhood of $x_{k}$ for all $1 \leq k \leq N$.

Assuming that $n \leq d$, we add $2(d-n)$ extra knots to $\pi$ to form the partition

$\tilde{\pi}: x_{-d+n}=\ldots=x_{-1}=x_{0}<x_{1} \leq \ldots \leq x_{N}<x_{N+1}=x_{N+2}=\ldots=x_{N+d+1-n}$. We now define the classical B-splines for the partition $\tilde{\pi}$ (see [4]):

$$
M_{k}(x) \equiv(d+1) g\left(x_{k}, x_{k+1}, \ldots, x_{k+d+1} ; x\right),-d+n \leq k \leq N-n,
$$

is $(d+1)$ times the $(d+1)$-th divided difference in $y$ of the function $g(y ; x) \equiv(y-x)_{+}^{d}$ based on the points $x_{k}, x_{k+1}, \ldots, x_{k+d+1}$. The normalized B-splines are defined by

$$
\psi_{k}(x) \equiv \frac{x_{k+d+1}{ }^{-x_{k}}}{d+1} M_{k}(x), \quad-d+n \leq k \leq N-n .
$$

It can be shown that $\left\{\psi_{k}(x)\right\}_{k=-d+n}^{N-n}$ form a basis for $S p_{0}(d, \pi)$ (see [4]).

The following lemma is a simple consequence of a theorem in [2].

LEMMA 1. For an arbitrary $(d+1)$-extended partition $\pi$, there exists a positive constant $D$ depending on $d$ but not on $\pi$ such that.

$$
\left\|\sum_{k=-d+n}^{N-\dot{n}} a_{k+d+1-n}\left(\frac{d+1}{x_{k+d+1}-x_{k}}\right)^{\frac{3}{2}} \psi_{k}\right\|_{L^{2}} \geq D\|\mathrm{a}\|_{2}
$$


for all a $\in R^{N+d+1-2 n}$ where $\|\mathrm{a}\|_{2} \equiv\left(\sum_{i=1}^{N+d+1-2 n} a_{i}^{2}\right)^{\frac{2}{2}}$.

We consider the case where the approximating subspace $S_{m} \equiv S p_{0}(d, \pi), m=N+d+1-2 n$, and the coordinate functions $\phi_{i}(x) \equiv \psi_{i+n-d-1}(x), \quad i=1,2, \ldots, m$. Assume that $f_{k} \leq d+1-n$ for all $i \leq k \leq N$ to ensure that $S_{0}(d, \pi) \subset W_{0}^{n, 2}[0,1]$. The spectral condition number of the Rayleigh-Ritz-Galerkin matrix $R$ is defined by

$$
\kappa(R) \equiv\|R\|_{2}\left\|R^{-1}\right\|_{2} \text { where }\|R\|_{2} \equiv \sup _{\mathrm{a} \in R^{m}}\|R \mathrm{a}\|_{2} /\|\mathrm{a}\|_{2} .
$$

Using (3), it can easily be shown that $R$ is positive definite and symmetric, and hence $\kappa(R)=\lambda^{-1} \Lambda$ where $\lambda$ and $\Lambda$ are the minimum and maximum eigenvalues, respectively, of $R$. The following theorem is analogous to that of Schultz [7].

THEOREM 1. If (3) holds and $\pi$ is an arbitrary $(d+1)$-extended partition of $[0,1]$ such that $f_{k} \leq d+1-n$ for all $1 \leq k \leq N$, then there exists a positive constant $C$ depending on $d$ but not on $\pi$ such that

$$
\kappa(R) \leq C(\Delta / \delta) \delta^{-2 n}
$$

Proof. From (4) and (3), we obtain for all $a \in R^{m}$,

$$
\mathrm{a}^{T} R \mathrm{a}=\int_{0}^{1}\left\{\sum_{j=0}^{n} p_{j}(x)\left[D^{j} \sum_{i=1}^{m} a_{i} \psi_{i+n-d-1}(x)\right]^{2}\right\} d x \geq K\left\|\sum_{i=1}^{m} a_{i} \psi_{i+n-d-1}\right\|_{L^{2}}^{2}
$$

which, by Lemma 1, yields

$$
\begin{aligned}
\mathrm{a}^{T} R \mathrm{a} & \geq K D^{2} \sum_{i=1}^{m} a_{i}^{2} \frac{\left(x_{i+n} x_{i+n-d-1}\right)}{d+1} \\
& \geq K D^{2}(d+1)^{-1} \delta\|\mathrm{a}\|_{2}^{2},
\end{aligned}
$$

and thus

$$
\lambda \geq K D^{2}(d+1)^{-1} \delta
$$


Conversely, since $p_{j}(x), 0 \leq j \leq n$, are bounded on $[0,1]$, there exists a positive constant $P$ such that, for all a $\in R^{m}$,

$$
\begin{aligned}
\mathrm{a}^{T} R \mathrm{a} & \leq P \sum_{j=0}^{n} \int_{0}^{1}\left[\sum_{i=1}^{m} a_{i} D^{j} \psi_{i+n-d-1}(x)\right]^{2} d x \\
& \leq P \sum_{j=0}^{n}(2 d+1) \sum_{i=1}^{m} a_{i}^{2} \int_{0}^{1}\left[D^{j} \psi_{i+n-d-1}(x)\right]^{2} d x,
\end{aligned}
$$

since $\psi_{i+n-d-1}(x), 1 \leq i \leq m$, has support $\left[x_{i+n-d-1}, x_{i+n}\right]$. Thus

$$
\mathrm{a}^{T} R \mathrm{a} \leq P(2 d+1) \sum_{i=1}^{m} a_{i}^{2}\left(x_{i+n}-x_{i+n-d-1}\right) \sum_{j=0}^{n}\left\|D^{j} \psi_{i+n-d-1}\right\|_{L^{\infty}}^{2} .
$$

Using Lemma 3.1 of [3], it can be shown that there exists a positive constant $E$ depending on $d$ but not on $\pi$ such that

$$
\sum_{j=0}^{n}\left\|D^{j} \psi_{i+n-d-1}\right\|_{L}^{2} \leq E \delta^{-2 n} \text { for all } 1 \leq i \leq m
$$

Hence

$$
\mathrm{a}^{T} R \mathrm{a} \leq P E(2 d+1)(d+1) \Delta \delta^{-2 n}\|\mathrm{a}\|_{2}^{2},
$$

and thus

$$
\Lambda \leq P E(2 d+1)(d+1) \Delta \delta^{-2 n} \text {. }
$$

Combining (9) and (10), we obtain the desired result with $C=P E(2 d+1)(d+1)^{2} K^{-1} D^{-2}$. //

A corollary analogous to the Corollary of [7] is clearly valid.

\section{Extension to higher dimensions}

We consider the following class of linear, self-adjoint, boundary value problems defined on an $M$-dimensional hypercube $\Omega \equiv \underset{j=1}{M}[0,1]$ with boundary $\partial \Omega$ :

$$
L[u(x)]=f(x), x \in \Omega, f \in L^{2}(\Omega),
$$


with homogeneous Dirichlet boundary conditions

$$
D^{\alpha} u(x)=0, \quad x \in \partial \Omega, \quad 0 \leq|\alpha| \leq n-1, n \geq 1,
$$

where the linear differential operator $L$ is defined by

$$
\left.L[u(x)] \equiv \sum_{0 \leq|\alpha|,|\beta| \leq n}(-1)^{|\alpha|}\right|_{D^{\alpha}}\left[p_{\alpha \beta}(x)_{D}^{\beta} u(x)\right] \text {. }
$$

We are using the usual multi-index notation, see [5]. Assume that all the coefficients $p_{\alpha \beta}(x)$ are bounded measurable functions in $\Omega$ and that $p_{\alpha \beta}=p_{\beta \alpha}$ for all $0 \leq|\alpha|,|\beta| \leq n$.

Let $W_{0}^{n, 2}(\Omega)$ denote the completion of the set of all $C^{\infty}(\bar{\Omega})$ functions having compact support in $\Omega$., with respect to the Sobolev norm

$$
\|w\|_{w^{n, 2}} \equiv\left\{\sum_{0 \leq|\alpha| \leq n} \int_{\Omega}\left[D^{\alpha} w(x)\right]^{2} d x\right\}^{\frac{1}{2}} .
$$

We assume that there exists a positive constant $K$ such that for all $w \in w_{0}^{n, 2}(\Omega)$,

$$
K\left\|_{w}\right\|_{L}^{2} \leq \int_{\Omega}\left\{\sum_{0 \leq|\alpha|,|\beta| \leq n} p_{\alpha \beta}(x) D^{\alpha} w(x) D^{\beta} w(x)\right\} d x .
$$

It can be shown that the problem (11)-(13) has a unique generalized solution and that the Rayleigh-Ritz method is applicable, see [6]. Let $\left\{\phi_{i}(x)\right\}_{i=1}^{m}$ be $m$ linearly independent coordinate functions such that $\phi_{i} \in W_{0}^{n, 2}(\Omega)$ for all $1 \leq i \leq m$. The Rayleigh-Ritz-Galerkin matrix $R \equiv\left(r_{i k}\right)$ for the problem $(11)-(13)$ is given by

(15) $r_{i k}=\int_{\Omega}\left\{\sum_{0 \leq|\alpha|,|\beta| \leq n} p_{\alpha \beta}(x) D^{\alpha} \phi_{k}(x) D^{\beta} \phi_{i}(x)\right\} d x, 1 \leq i, k \leq m$.

For each $j, 1 \leq j \leq M$, let $\pi_{j}$ be a $(d+1)$-extended partition of $[0,1]$ in the $j$-th dimension:

$$
\pi_{j}: 0=x_{0}^{(j)}<x_{1}^{(j)} \leq x_{2}^{(j)} \leq \ldots \leq x_{N_{j}}^{(j)}<x_{N_{j}+1}^{(j)}=1
$$


and let $\Delta_{j}$ and $\delta_{j}$ be defined as in (5). Using expression (6), we construct the normalized $B$-spline basis $\left\{\psi_{k}\left(x^{(j)}\right)\right\}_{k=-d+n}^{N-n}$ for $S p_{0}\left(d, \pi_{j}\right), j=1,2, \ldots, M$. Let $\bar{\pi} \equiv \underset{j=1}{M} \pi_{j}$ be a $(d+1)$-extended product partition of $\Omega$ and let

$$
\bar{\Delta} \equiv \max _{1 \leq j \leq M} \Delta_{j} \text { and } \bar{\delta} \equiv \min _{1 \leq j \leq M} \delta_{j} .
$$

The extended multivariate spline space $S p_{0}(d, \bar{\pi})$ is defined to be the tensor product $\bigotimes_{j=1}^{M} S p_{0}\left(d, \pi_{j}\right)$. It can be shown that $S p_{0}(d, \bar{\pi})$ is the linear span of all the normalized multivariate B-splines $\psi_{k_{1}}\left(x^{(1)}\right) \psi_{k_{2}}\left(x^{(2)}\right) \ldots \psi_{k_{M}}\left(x^{(M)}\right),-d+n \leq k_{j} \leq N_{j}-n$, $j=1,2, \ldots, M$

which we rename as $\left\{B_{i}(x)\right\}_{i=1}^{m}$, where

$$
\mathrm{x}=\left(x^{(1)}, x^{(2)}, \ldots, x^{(M)}\right) \text { and } m=\prod_{j=1}^{M}\left(N_{j}+d+1-2 n\right) .
$$

Using Lemma 1 , it is straightforward to prove the following:

LEMMA 2. For an arbitrary $(d+1)$-extended product partition $\bar{\pi}$ of $\Omega$, there exists a positive constant $\bar{D}$ depending only on $d$ such that for azz a $\in R^{m}$,

$$
\left\|\sum_{i=1}^{m} a_{i} B_{i}\right\|_{L^{2}} \geq \bar{D} \bar{\delta}^{M / 2}\|\mathrm{a}\|_{2} .
$$

In applying the Rayleigh-Ritz method, let the approximating subspace $S_{m} \equiv S p_{0}(d, \bar{\pi})$ and let the coordinate functions $\phi_{i}(\mathrm{x}) \equiv B_{i}(\mathrm{x})$, $i=1,2, \ldots, m$. Assuming that the maximum multiplicity of the interior knots of $\pi_{j}$ is less than or equal to $d+1-n$, for all $1 \leq j \leq M$, then it can be shown that $s p_{0}(d, \bar{\pi}) \subset w_{0}^{n, 2}(\Omega)$, see [6]. 
THEOREM 2. If (14) holds and $\bar{\pi}$ is an arbitrary $(d+1)$-extended product partition of $\Omega$ such that the multiplicity assumption above is valid, then there exists a positive constant $\bar{c}$ depending only on $d$ such that

$$
\kappa(R) \leq \bar{C}(\bar{\Delta} / \bar{\delta})^{M_{\delta}-2 n}
$$

Proof. From (15) and (14), we obtain for all a $\in R^{m}$,

$$
\begin{aligned}
\mathrm{a}^{T} R \mathrm{a} & =\int_{\Omega}\left\{\sum_{0 \leq|\alpha|,|\beta| \leq n} p_{\alpha \beta}(\mathrm{x}) D^{\alpha}\left[\sum_{i=1}^{m} a_{i} B_{i}(\mathrm{x})\right] D^{\beta}\left[\sum_{i=1}^{m} a_{i} B_{i}(\mathrm{x})\right]\right\} d \mathrm{x} \\
& \geq K\left\|\sum_{i=1}^{m} a_{i} B_{i}\right\|_{L^{2}}^{2},
\end{aligned}
$$

which, by Lemma 2, yields

$$
\mathrm{a}^{T} R \mathrm{a} \geq K \bar{D}^{2} \bar{\delta}^{M}\|\mathrm{a}\|_{2}^{2}
$$

and thus

$$
\lambda \geq K \vec{D}^{2} \delta^{M} .
$$

Conversely, since $p_{\alpha \beta}(x), 0 \leq|\alpha|,|\beta| \leq n$, are bounded in $\Omega$, there exists a positive constant $Q$ such that

$$
\mathrm{a}^{T} R \mathrm{a} \leq Q \sum_{0 \leq|\alpha| \leq n} \int_{\Omega}\left[D^{\alpha} \sum_{i=1}^{m} a_{i} B_{i}(\mathrm{x})\right]^{2} d \mathrm{x},
$$

and using the minimal support properties of $\left\{B_{i}\right\}_{i=1}^{m}$, it can be shown that there exists a positive constant $F$ depending only on $d$ such that

$$
\begin{aligned}
\mathrm{a}^{T} R \mathrm{a} & \leq Q F \sum_{0 \leq|\alpha| \leq n} \sum_{i=1}^{m} a_{i}^{2} \int_{\Omega}\left[D^{\left.\alpha_{B}(\mathrm{x})\right]^{2}} d \mathrm{x}\right. \\
& \leq Q F \sum_{i=1}^{m} a_{i}^{2}(d+1)^{M} \bar{\Delta}^{M} \sum_{0 \leq|\alpha| \leq n}\left\|D^{\alpha} B_{i}\right\|_{L^{\infty}}^{2} .
\end{aligned}
$$

Using Lemma 3.1 of [3], it can be shown that there exists a positive constant $\bar{E}$ depending only on $d$ such that 


$$
\sum_{0 \leq|\alpha| \leq n}\left\|D^{\alpha_{B}}\right\|_{L^{\infty}}^{2} \leq E \delta^{-2 n} \text { for all } 1 \leq i \leq m \text {. }
$$

Hence

$$
\mathrm{a}^{T} R \mathrm{a} \leq Q \bar{Q} \bar{E}(d+1)^{M-M} \bar{\Delta}^{-2 n}\|\mathrm{a}\|_{2}^{2}
$$

and thus

$$
\Lambda \leq Q \bar{F} \bar{E}(d+1)^{M-\Delta^{M}} \bar{\delta}^{-2 n}
$$

Combining (18) and (19), we obtain the desired result with $\bar{C}=Q \bar{E} \bar{E}(d+1)^{M} K^{-1} \bar{D}^{-2}$

\section{References}

[1] Carl de Boor, "On uniform approximation by splines", J. Approximation Theory 1 (1968), 219-235.

[2] Carl de Boor, "The quasi-interpolant as a tool in elementary polynomial spline theory", Approximation theory, 269-276 (Proc. Internat. Sympos., Univ. Texas, Austin, Texas, 1973. Academic Press, New York, London, 1973).

[3] C. de Boor and G.J. Fix, "Spline approximation by quasiinterpolants", J. Approximation Theory 8 (1973), 19-45.

[4] H.B. Curry and 1.J. Schoenberg, "On Pólya frequency functions IV: the fundamental spline functions and their limits", J. Analyse Math. 17 (1966), 71-107.

[5] С.Г. Михлин, Чнсленная реализацня вариационных методов (Izdat. "Nauka", Moscow, 1966).

S.G. Mikhlin, The numerical performance of variational methods (translated by R.S. Anderssen. Wolters-Noordhoff, Groningen, 1971).

[6] Bernard J. Omodei, "Stability of the Rayleigh-Ritz-Galerkin procedure for elliptic boundary value problems" ( $\mathrm{PhD}$ thesis, Australian National University, Canberra, 1976). See also: Abstract, Bulz. Austral. Math. Soc. 14 (1976), 471-472. 
[7] Martin H. Schultz, "The condition number of a class of Rayleigh-RitzGalerkin matrices", Bull. Amer. Math. Soc. 14 (1970), 840-844.

Department of Mathematics,

University of Manchester,

Manchester,

England.

Present Address:

School of Mathematical Sciences,

Flinders University of South Australia,

Bedford Park,

South Australia. 